\title{
Resposta de plantas de alho livres de vírus ao nitrogênio em ambiente protegido
}

\author{
Lucilene JC Fernandes ${ }^{1,3}$; Leonardo T Büll' ${ }^{1}$ Juliano C Corrêa ${ }^{1}$; Marcelo A Pavan ${ }^{1}$; Isao Imaizumi ${ }^{2}$ \\ ${ }^{1}$ UNESP-FCA-Faz. Exp. Lageado, C. Postal 237, 18603-970 Botucatu-SP; ${ }^{2}$ Genove Agronegócios, Rod. BR 452, km 225, C. Postal 27, \\ 38175-000 Santa Juliana-MG; ${ }^{3}$ Bolsista CAPES; ljcfernandes@ig.com.br
}

\section{RESUMO}

A adubação nitrogenada associada ao uso de bulbos isentos de vírus contribui para o aumento de produtividade e qualidade do alho, fato que pode incentivar o produtor rural a investir na cultura, pois garante maior retorno econômico. O objetivo do trabalho foi avaliar o índice relativo de clorofila, estado nutricional, a produção total e comercial do alho livre de vírus e a correlação entre essas variáveis em função de doses de nitrogênio. $\mathrm{O}$ experimento foi instalado sob condições de ambiente protegido de maio a setembro de 2006, utilizando o delineamento em blocos casualizados, com seis repetições. Os tratamentos consistiram da aplicação em cobertura de doses crescentes de $\mathrm{N}\left(0,20,40,80,160\right.$ e $\left.320 \mathrm{~kg} \mathrm{ha}^{-1}\right)$, utilizando como fonte o nitrato de amônio, na proporção de $25 \%$ da dose no estádio de 3 a 4 folhas, $25 \%$ antes da formação dos bulbilhos e $50 \%$ após a diferenciação. $\mathrm{O}$ aumento das doses de $\mathrm{N}$ resultou em maior índice relativo de clorofila, aumentou a absorção de $\mathrm{N}$ e reduziu absorção de $\mathrm{P} \mathrm{e} \mathrm{K}$ nas folhas, proporcionou maior produtividade total e comercial, sem que ocorresse pseudoperfilhamento.

Palavras-chave: Allium sativum, clorofilômetro, nutrição.

\begin{abstract}
Nitrogen fertilization in garlic free of virus cultivated in protected environment

Nitrogen fertilization associated to the utilization of bulb free of virus enhances garlic yield and quality and it can incentive growers to invest in the crop whereas it allows a better economic return. The aim of this research was to evaluate the relative chlorophyll index (RCI), plant nutrition and total and commercial garlic yield and verify the correlation among these variables in relation to nitrogen fertilization. The experiment was carried out under greenhouse from May to September 2006. The experimental design consisted of randomized blocks with six replications. The treatments were application of crescent levels of nitrogen corresponding to $0,20,40,80,160$, and $320 \mathrm{~kg} \mathrm{~N} \mathrm{ha}^{-1}$. The $\mathrm{N}$ source was ammonium nitrate; $25 \%$ of the dose was applied when plants presented 3 or 4 leaves, $25 \%$ when plants presented 5 or 6 leaves, and $50 \%$ after the differentiation. The application of crescent levels of nitrogen proportioned higher relative chlorophyll index $(\mathrm{RCI})$, increased the $\mathrm{N}$ content and reduced $\mathrm{P}$ and $\mathrm{K}$ content in leaves, enhanced total and commercial yield without secondary growth.
\end{abstract}

Keywords: Allium sativum, chlorophyll meter, nutrition.

(Recebido para publicação em 4 de novembro de 2008; aceito em 8 de fevereiro de 2010) (Received on November 4, 2008; accepted on February 8, 2010)

$\mathrm{O}$ principal fator que possibilita maiores rendimentos na cultura do alho (Allium sativum) é a aplicação de fertilizantes, em especial o nitrogênio, pois é o nutriente que mais contribui para o aumento da produtividade de bulbos por exercer efeito rápido e pronunciado sobre o desenvolvimento vegetal nessa cultura (Büll et al., 2002).

Repostas à adubação com $\mathrm{N}$ na cultura do alho foram obtidas desde a dose de $40 \mathrm{~kg} \mathrm{ha}^{-1}$ até a dose de $180 \mathrm{~kg} \mathrm{ha}^{-1}$ (Patel et al., 1996; Verma et al.,1996; Resende et al., 1993; Resende et al., 2000; Resende \& Souza, 2001a; Büll et al., 2002; Marouelli et al., 2002). Ao contrário, Costa et al. (1993), Lipinski et al. (1995) e Sadaria et al. (1997) não verificaram efeitos significativos do $\mathrm{N}$ na produtividade total e comercial do alho. Vale ressaltar que quando o $\mathrm{N}$ é aplicado em cobertura no período de bulbificação ocorre maior incidência de pseudoperfilhamento (Resende \&
Souza, 2001a,b; Büll et al., 2001, 2002).

Portanto, a dose de $\mathrm{N}$ em cobertura deve resultar na máxima produtividade, desde que aplicada na época correta para evitar o pseudoperfilhamento.

O alho é propagado assexuadamente, permitindo que patógenos sejam facilmente disseminados através das gerações, causando acúmulo de viroses e degenerescência dos clones. A técnica de cultura de tecidos possibilita a obtenção de plantas de alho isentas de patógenos, em especial os vírus, que condiciona respostas diferentes à adubação nitrogenada, quando comparadas com plantas obtidas convencionalmente. Assim, a associação da aplicação de fertilizantes à técnica de cultura de tecidos, possibilitará que estas plantas apresentem maior potencial produtivo em razão do aumento do peso e diâmetro de bulbos, refletindo em maior retorno econômico para o produtor (Resende et al., 2000).
Conduziu-se este trabalho com o objetivo de avaliar o índice relativo de clorofila, estado nutricional, produção total e comercial do alho livre de vírus e a correlação entre essas variáveis em função das doses de nitrogênio aplicadas.

\section{MATERIAL E MÉTODOS}

O experimento foi desenvolvido no município de Santa Juliana-MG, sob condições de ambiente protegido de 12/05/06 a 01/09/06. O solo, classificado como Latossolo Vermelho-Amarelo (Embrapa, 1999) foi amostrado na camada de 0-20 cm para realização da análise química (Raij et al., 2001), que apresentou os seguintes resultados: $\mathrm{pH}\left(\mathrm{CaCl}_{2}\right)=6,0 ;$ M.O. $=24 \mathrm{~g} \mathrm{~kg}^{-1}$; $\mathrm{P}(\mathrm{resina})=104 \mathrm{mg} \mathrm{dm}^{-3} ; \mathrm{H}+\mathrm{Al}=25$ $\mathrm{mmol}_{\mathrm{c}} \mathrm{dm}^{-3} ; \mathrm{K}=4 \mathrm{mmol}_{\mathrm{c}} \mathrm{dm}^{-3} ; \mathrm{Ca}=54$ $\mathrm{mmol}_{\mathrm{c}} \mathrm{dm}^{-3} ; \mathrm{Mg}=14 \mathrm{mmol}_{\mathrm{c}} \mathrm{dm}^{-3} ; \mathrm{CTC}=$ $97 \mathrm{mmol}_{\mathrm{c}} \mathrm{dm}^{-3} ; \mathrm{V}=74 \%$; $\mathrm{B}=0,44 \mathrm{mg}$ 
$\mathrm{dm}^{-3} ; \mathrm{Zn}=5,2 \mathrm{mg} \mathrm{dm}^{-3}$.

$\mathrm{O}$ delineamento experimental utilizado foi em blocos casualizados, com seis repetições. Os tratamentos consistiram de aplicações em cobertura com doses crescentes de $\mathrm{N}(0,20,40$, 80,160 e $320 \mathrm{~kg} \mathrm{~N} \mathrm{ha}^{-1}$ ), tendo como fonte o nitrato de amônio. Essas doses foram parceladas em razão do estádio fenológico da cultura, aplicando-se 25\% da dose quando as plantas apresentaram de 3 a 4 folhas, 25\% quando apresentaram de 5 a 6 folhas, o que ocorreu antes da diferenciação, e 50\% após a fase de diferenciação, estádio de início do crescimento dos bulbilhos.

As parcelas foram dimensionadas com 2,0 m de comprimento por 1,2 $\mathrm{m}$ de largura do canteiro, com espaçamento de $10 \mathrm{~cm}$ entre plantas e $20 \mathrm{~cm}$ entre linhas. A adubação de plantio foi ajustada para a camada de $0-20 \mathrm{~cm}$ e foram aplicados $680 \mathrm{~kg} \mathrm{ha}^{-1}$ de $\mathrm{P}_{2} \mathrm{O}_{5}$ antes da gradagem, sendo $1 / 3$ na forma de termofosfato e 2/3 de superfosfatosimples, $50 \mathrm{~kg} \mathrm{ha}^{-1}$ de $\mathrm{KCl}, 10 \mathrm{~kg} \mathrm{ha}^{-1}$ de ácido bórico e 10 $\mathrm{kg} \mathrm{ha}^{-1}$ de sulfato de zinco. Junto com a primeira adubação nitrogenada de cobertura foi adicionado o equivalente a $50 \mathrm{~kg} \mathrm{ha}^{-1}$ de $\mathrm{KCl}$.

Utilizou-se a cv. Caçador LV (livre de vírus), pertencente ao grupo dos "alhos nobres" e das cultivares tardias. Os bulbos caracterizam-se por apresentar bulbilhos graúdos e em pequeno número, coloração externa roxa e alta capacidade de conservação (Filgueira, 2000), sendo bem aceitos no mercado por ter qualidade comparável à do alho argentino. Os bulbilhos isentos de vírus foram obtidos através de cultura de tecidos e termoterapia, na UNESP-FCA, aclimatizados em casa-de-vegetação e multiplicados por quatro gerações em telado no município de GuarapuavaPR. Foram utilizados para o plantio bulbilhos pesando entre 3 e 4 gramas, de bulbos $n^{\circ} 6$ (diâmetro transversal maior que 47 até $56 \mathrm{~mm}$ ), de acordo com a classificação da Circular n ${ }^{\circ}$ 50/81 da Comissão Técnica de Normas e Padrões do Ministério da Agricultura.

A colheita ocorreu aos 112 dias após o plantio (DAP). Foram adotados os tratos culturais referentes à cultura do alho como medidas preventivas de controle de pragas e doenças.
As avaliações de índice relativo de clorofila (IRC) foram realizadas nos mesmos dias das aplicações dos tratamentos e 15 dias após a última aplicação das doses de N, amostrando-se 10 plantas por parcela. O IRC foi determinado com o auxílio do medidor portátil, o clorofilômetro, modelo SPAD-502 da Minolta Corporation Ltda, amostrandose a parte central da folha recentemente expandida e fisiologicamente madura.

As folhas em que foram realizadas as leituras de IRC, à exceção da primeira época de avaliação, foram coletadas para análise química dos teores foliares de macronutrientes, segundo metodologia descrita por Malavolta et al. (1997).

As plantas destinadas à avaliação da produção foram coletadas a partir de $0,5 \mathrm{~m}$ de cada extremidade da parcela, coletando 10 plantas por linha, totalizando 50 plantas por parcela. Após a colheita, as plantas passaram pelo período de cura por 30 dias, a fim de favorecer gradual perda de umidade e concentração de sólidos nos bulbos, melhorando a conservação (Filgueira, 2000). Após esse intervalo, os bulbos foram classificados em função do diâmetro transversal em classes de 3 a 7 , segundo a Comissão Técnica de Normas e Padrões do Ministério da Agricultura

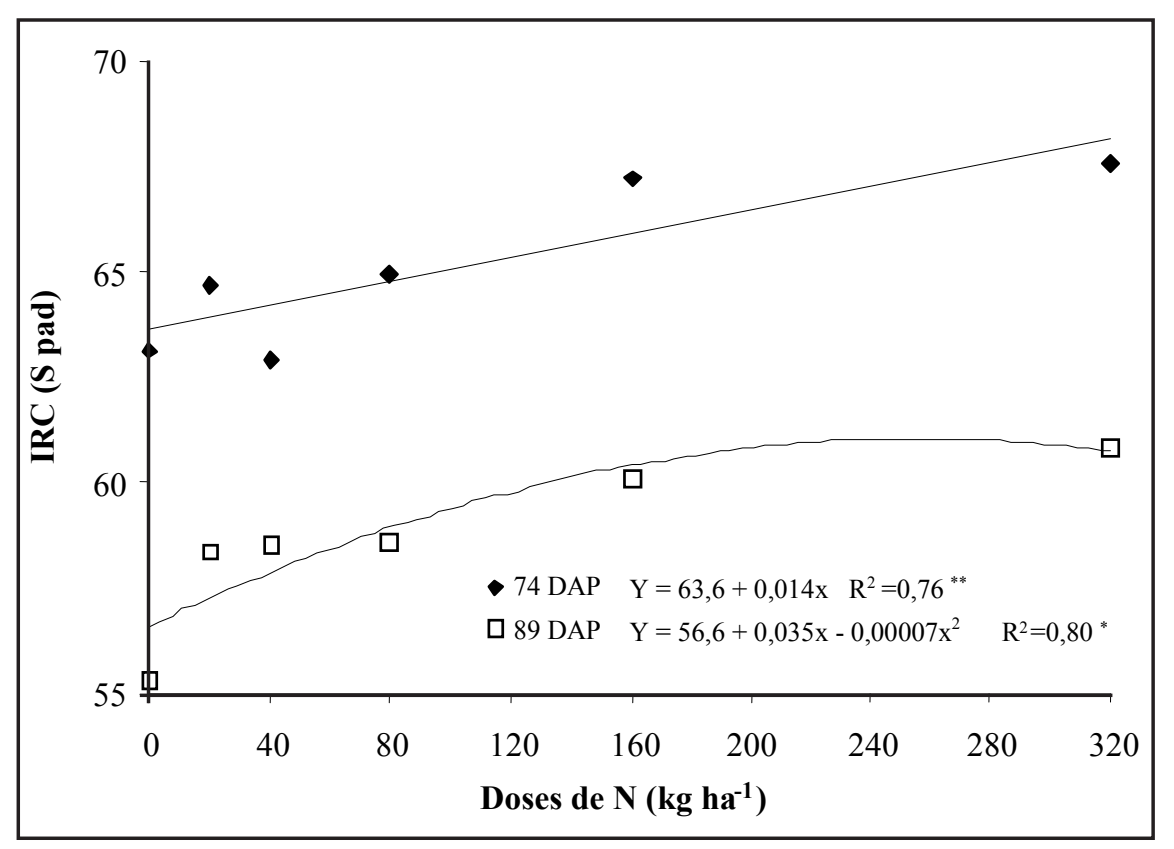

Figura 1. Índice Relativo de Clorofila (IRC) em folhas de plantas de alho, na fase de diferenciação (74 DAP) e 15 dias após (89 DAP) em função de doses de nitrogênio (relative chlorophyll index (RCI) in leaves of garlic plants, at differentiation (74 DAP) and 15 days after (89 DAP) in response to the nitrogen rates). Santa Juliana, GENOVE, 2006. 
nológicos de diferenciação (74 DAP) e 15 dias após (89 DAP) (Figura 1). Não houve, no entanto, efeito significativo para essa variável quando a amostragem foi realizada nos estádios de 3 a 4 folhas (13 DAP) e de 5 a 6 folhas (28 DAP). Esse resultado pode ser justificado em razão do $\mathrm{N}$ ser componente do grupo pirrólico na molécula de clorofila, ou seja, seu fornecimento em função das doses estaria contribuindo na formação dessa molécula e, consequentemente, aumentando os valores de IRC (Lima, 2005).

Os maiores valores de IRC foram obtidos nas doses de $320 \mathrm{~kg} \mathrm{ha}^{-1}$ de $\mathrm{N}$ quando a folha de alho foi amostrada no estádio de diferenciação e de 250 $\mathrm{kg} \mathrm{ha}^{-1}$ de $\mathrm{N}$ quando a folha foi amostra 15 dias após a diferenciação. Resende (1994) sugeriu o período de 6 a 9 dias como intervalo para se obter o máximo de aumento do teor de clorofila devido à aplicação de N. Entretanto, Büll et al. (2002) não verificaram aumento no teor de clorofila em função de doses crescentes de $\mathrm{N}$, utilizando-se a cultivar Caçador, com bulbilhos sem a utilização da técnica de cultura de tecidos.

As plantas de alho absorveram mais $\mathrm{N}$ e menos $\mathrm{P}$ e K em função do aumento das doses de N (Figura 2), com a amostragem das plantas realizada no mesmo estádio da variável IRC. Os teores médios de $\mathrm{N}$ aumentaram de acordo com as doses desse nutriente aplicado, sendo que o maior teor de $\mathrm{N}$ na folha de alho foi observado na dose de $320 \mathrm{~kg} \mathrm{ha}^{-1} \mathrm{de}$ N. De acordo com Raij et al. (1996), as faixas de suficiência para N, P e K

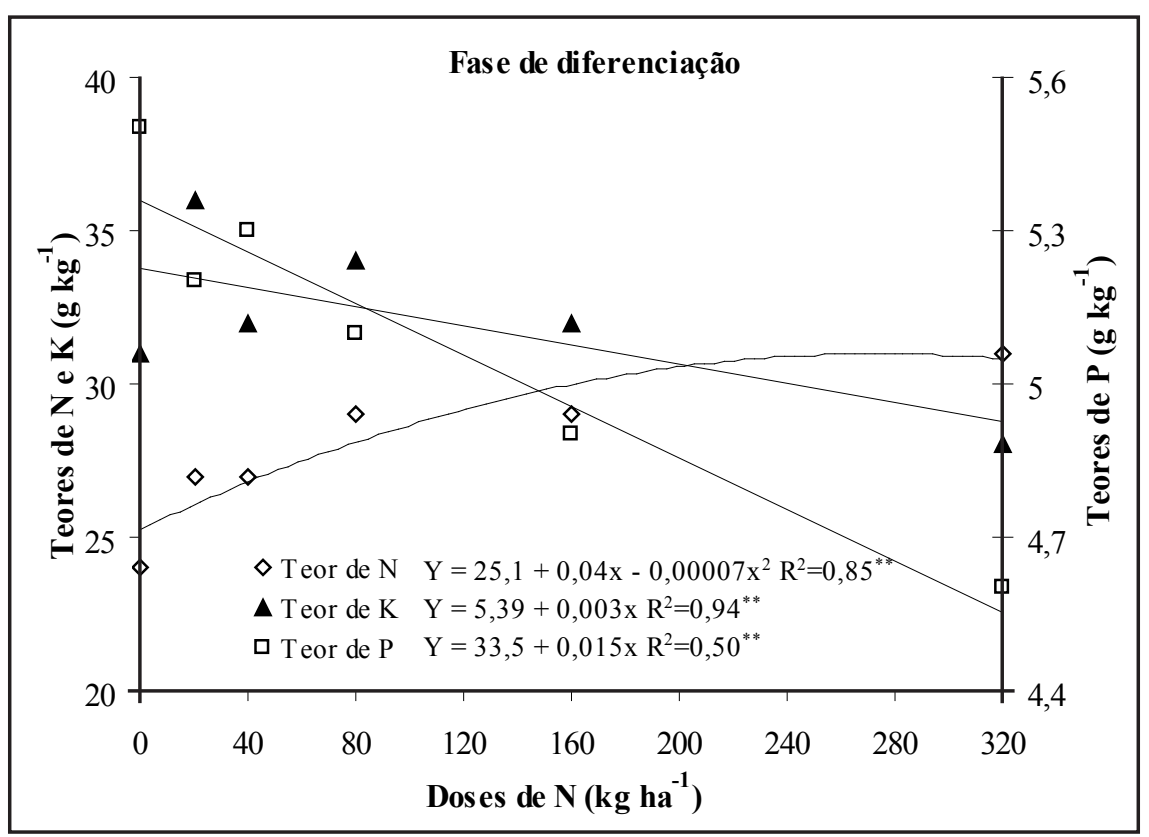

Figura 2. Teores foilares de N, P e K, na fase de diferenciação (74 DAP), em plantas de alho, em função de doses de nitrogênio (leaves concentrations of N, P and K, at differentiation (74 DAP), in garlic plants, in response to the nitrogen rates). Santa Juliana, GENOVE, 2006.

consideradas adequadas para a cultura do alho, apresentam valores entre 25 a $30 \mathrm{~g} \mathrm{~kg}^{-1}$ de N, 3,0 a 5,0 $\mathrm{g} \mathrm{kg}^{-1}$ de P e de 30 a $44 \mathrm{~g} \mathrm{~kg}^{-1}$ de K. Assim, os teores de $\mathrm{N}, \mathrm{P}$ e K obtidos podem ser considerados adequados, pois estão dentro ou próximos da faixa de suficiência considerada adequada à cultura, tendo como principais parâmetros o desenvolvimento da planta e a produtividade alcançada.

$\mathrm{A}$ redução nos teores de $\mathrm{P}$ e $\mathrm{K}$ nas folhas de alho em função das doses crescentes de N (Figura 2) pode ser justificada em razão das maiores doses de $\mathrm{N}$ terem proporcionado maiores bulbilhos, causando o efeito de diluição para esses

Tabela 1. Distribuição dos bulbos (\%), nas classes $<3,3,4,5$, e 6 , em função de doses crescentes de $\mathrm{N}\left(\mathrm{kg} \mathrm{ha}^{-1}\right)$ (distribution of bulbs (\%), in the class $<3,3,4,5$, and 6 , in response to the nitrogen rates $\left.\left(\mathrm{kg} \mathrm{ha}^{-1}\right)\right)$. Santa Juliana, GENOVE, 2006.

\begin{tabular}{lccccc}
\hline \multirow{2}{*}{ Doses de N } & \multicolumn{5}{c}{ Classes } \\
\cline { 2 - 6 } $\mathbf{0}$ & $<\mathbf{3}$ & $\mathbf{3}$ & $\mathbf{4}$ & $\mathbf{5}$ & $\mathbf{6}$ \\
$\mathbf{2 0}$ & 5 & 26 & 44 & 22 & 3 \\
$\mathbf{4 0}$ & 4 & 16 & 43 & 30 & 6 \\
$\mathbf{8 0}$ & 3 & 25 & 36 & 33 & 3 \\
$\mathbf{1 6 0}$ & 4 & 15 & 39 & 30 & 13 \\
$\mathbf{3 2 0}$ & 2 & 12 & 35 & 35 & 16 \\
\hline & 2 & 9 & 27 & 39 & 23 \\
\hline
\end{tabular}

ns: não significativo; ${ }^{* *}$ significativo a $1 \%$ pelo teste $\mathrm{F}$; L: equação linear (ns: not significant, ** $1 \%$ significant by $\mathrm{F}$ test; L: linear equation) nutrientes, pois no estádio de enchimento dos bulbilhos, o dreno mais forte passa a ser esse órgão, portanto deve existir redistribuição mais eficiente do $\mathrm{P}$ e $\mathrm{K}$ no sentido folha - bulbilhos, principalmente, quando os bulbilhos são maiores. É nesse órgão que está havendo maior gasto energético, bem como a absorção e transporte dos direcionados, preferencialmente, para os bulbilhos. Tanto o $\mathrm{P}$ como o K, são nutrientes móveis dentro da planta, sendo muito exigidos nos locais de maior crescimento, no caso o bulbilho, pois estão envolvidos fisiologicamente com as funções de transporte de energia (ATP) e o desencadeamento das atividades enzimáticas, entre outras (Taiz \& Zeiger, 2004).

A adubação nitrogenada em cobertura proporcionou maior produtividade total e comercial, as quais apresentaram comportamento linear crescente com o aumento das doses de N (Figura 3), sendo que a dose de $320 \mathrm{~kg} \mathrm{ha}^{-1}$ de $\mathrm{N}$ proporcionou valores de $9,1 \mathrm{t} \mathrm{ha}^{-1}$ para a produtividade total e de $9,0 \mathrm{t} \mathrm{ha}^{-1}$ para a comercial, respectivamente. Efeitos significativos da adubação com $\mathrm{N}$ foram obtidos até a dose de $97 \mathrm{~kg} \mathrm{~N} \mathrm{ha}^{-1}$ por Marouelli et al. (2002), de $149 \mathrm{~kg} \mathrm{~N}$ $\mathrm{ha}^{-1}$ por Resende \& Souza (2001a), e de $160 \mathrm{~kg} \mathrm{~N} \mathrm{ha}^{-1}$ por Büll et al., (2002). Ao contrário, Costa et al. (1993) não 


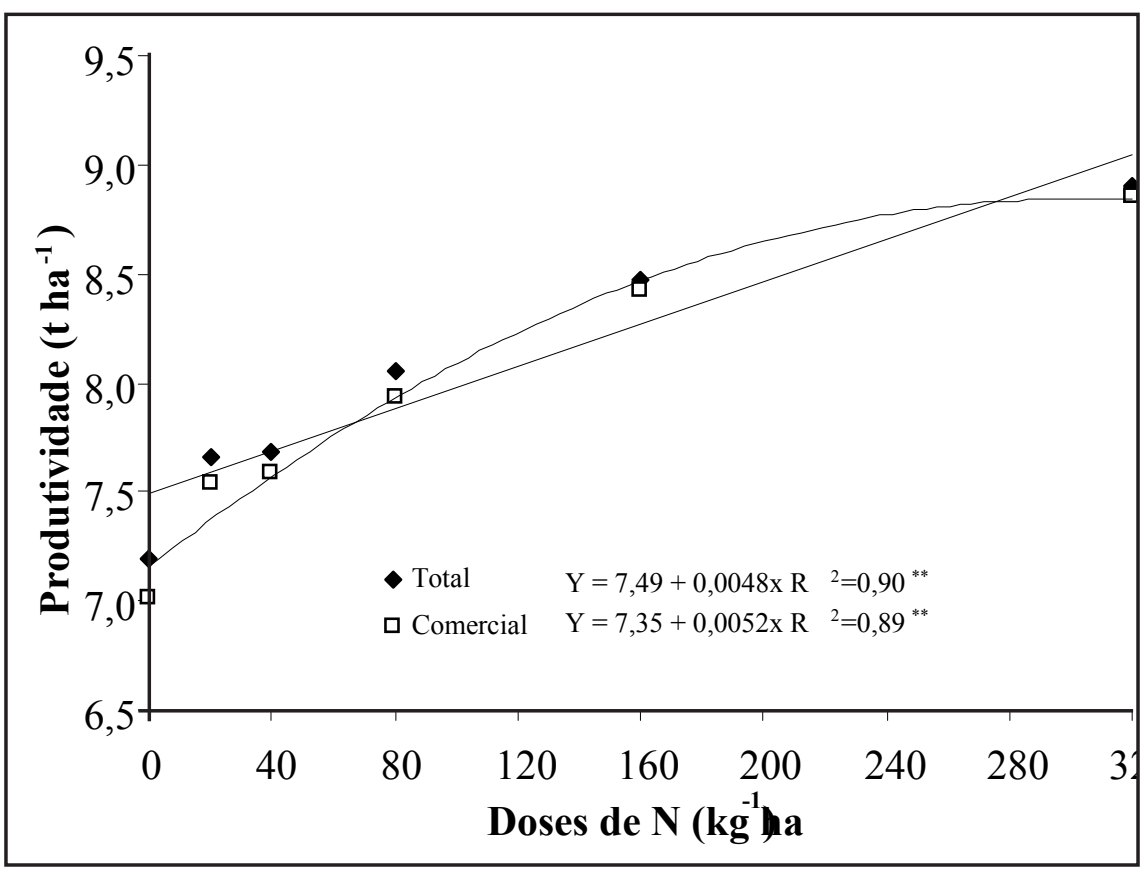

Figura 3. Produtividades total e comercial $\left(\mathrm{t} \mathrm{ha}^{-1}\right)$ de bulbos de alho, em função de doses de nitrogênio (total and commercial yield $\left(\mathrm{t} \mathrm{ha}^{-1}\right)$ of garlic bulbs, in response to the nitrogen rates). Santa Juliana, GENOVE, 2006.

Tabela 2. Coeficiente de correlação entre índice relativo de clorofila (IRC), teor de nitrogênio na folha e produtividades total e comercial (coefficient of correlation between relative chlorophyll index (RCI), nitrogen concentration in leaves, and total and commercial yield. Santa Juliana, GENOVE, 2006.

\begin{tabular}{llc}
\hline Fatores correlacionados & Fase de amostragem & $\begin{array}{c}\text { Coeficiente de } \\
\text { correlação }\end{array}$ \\
\hline \multirow{2}{*}{ IRC x N foliar } & 5 a 6 folhas & $\mathrm{ns}$ \\
& Diferenciação & $0,73^{* *}$ \\
& 15 dias após diferenciação & $0,80^{* *}$ \\
\hline IRC x Produtividade total & 5 a 6 folhas & $\mathrm{ns}$ \\
& Diferenciação & $0,49^{* *}$ \\
\hline \multirow{2}{*}{ IRC x Produtividade comercial } & Diferenciação & $0,60^{* *}$ \\
\hline & 15 dias após diferenciação & $\mathrm{ns}$ \\
\hline \multirow{2}{*}{ N foliar x Produtividade total diferenciação } & Diferenciação & $0,57^{* *}$ \\
\hline & 15 dias após diferenciação & $0,47^{* *}$ \\
\hline N foliar x Produtividade com- & 5 a 6 folhas & $\mathrm{ns}$ \\
ercial & Diferenciação & $\mathrm{ns}$ \\
& 15 dias após diferenciação & $0,47^{* *}$ \\
\hline
\end{tabular}

ns: não significativo; **significativo a $1 \%$ pela correlação de Pearson (ns: not significant; $* * 1 \%$ significant by Pearson correlation). verificaram efeito significativo do $\mathrm{N}$ na produtividade total e comercial do alho quando utilizaram até $120 \mathrm{~kg} \mathrm{ha}^{-1}$, assim como, Lipinski et al. (1995), até dose de $240 \mathrm{~kg} \mathrm{ha}^{-1}$ de N e Sadaria et al. (1997) quando foram aplicados até $75 \mathrm{~kg} \mathrm{ha}^{-1} \mathrm{de}$ $\mathrm{N}$. Vale ressaltar, que esse experimento foi plantado com bulbos provenientes de cultura de tecido, portanto, livres de vírus e em condições de ambiente protegido e irrigação por gotejamento, fatores que contribuíram para a resposta positiva das plantas de alho ao $\mathrm{N}$.

A adubação nitrogenada aumentou o diâmetro dos bulbos das classes 5 e 6 , e reduziu os da classe 3 e 4 . Não houve efeito significativo para as classes $<3$ (Tabela 1). Esse fato deve ser destacado, pois bulbos das classes 5 e 6 são maiores que os da classes 3 e 4, apresentando maior valor comercial e preferência pelo consumidor. Vale ressaltar que a disponibilidade de $\mathrm{N}$ no solo exerce influência relevante sobre o valor nutritivo do alimento, sendo considerado o macronutriente que proporciona qualidade ao produto, por fazer parte de compostos como aminoácidos e proteínas (Malavolta, 1996).

As correlações entre o índice relativo de clorofila (IRC), teor de nitrogênio na folha e produtividades total e comercial foram significativas quando as plantas de alho se encontravam nos estádios de diferenciação e 15 dias após essa fase, não havendo efeito significativo entre essas variáveis quando as plantas estavam no início do desenvolvimento, ou seja, no estádio de 5 a 6 folhas (Tabela 2).

A correlação positiva entre o IRC e $\mathrm{N}$ foliar $\left(0,73^{* *}\right.$ e $\left.0,80^{* *}\right)$ pode ser explicada pela maior quantidade de clorofila em função do maior teor de N, uma vez que esse nutriente participa com 4 moléculas na sua composição química (Taiz \& Zeiger, 2004). Dados semelhantes foram obtidos por Lima (2005), que avaliou a correlação entre IRC e N foliar a cada 10 dias durante o ciclo da cultura de 130 dias, utilizando a cultivar Caçador, obtendo o maior coeficiente de correlação $\left(0,76^{* *}\right)$ aos 70 dias após a emergência das plantas.

Houve correlação positiva, também, entre o IRC e as variáveis produtividade total $\left(0,49^{* *}\right.$ e $\left.0,60^{* *}\right)$ e comercial 
$(0,48 * *$ e $0,57 * *)$, permitindo estimar a produtividade em função das leituras IRC, quando as plantas forem amostradas no estádio de diferenciação ou após 15 dias a diferenciação (Tabela 2). As produtividades total e comercial também se correlacionaram positivamente com o teor de $\mathrm{N}$ nas folhas $(0,47 * *$ e $0,47 * *$, respectivamente), mostrando que os teores de nitrogênio, até a concentração de $31 \mathrm{~g} \mathrm{~kg}^{-1}$ nas folhas de alho, amostradas no estádio de diferenciação floral ou 15 dias após esta fase, condicionam maior produtividade total e comercial às plantas de alho.

Não foram obtidas plantas pseudoperfilhadas, devido, provavelmente, à suspensão da irrigação, no período que antecedeu a diferenciação, e também à cobertura com $50 \%$ da dose somente após a diferenciação, práticas essas utilizadas por produtores de alho para evitar o surgimento dessa anomalia. A ocorrência de pseudoperfilhamento está associada, dentre outros fatores, à disponibilidade de água no solo e à adubação nitrogenada próxima à fase de diferenciação. Assim, quanto maior a disponibilidade de água no solo e mais próxima a adubação de cobertura da diferenciação, maior é a porcentagem de plantas pseudoperfilhadas. Portanto, recomenda-se que manejos como a adubação de cobertura com N e a irrigação sejam realizados no estádio adequado de desenvolvimento da cultura, permitindo que essa anomalia seja minimizada ou até eliminada, como foi o caso desse experimento.

Pode-se concluir que, nas condições deste experimento, a melhor dose para a produção de clorofila foi de $320 \mathrm{~kg}$ de $\mathrm{N} \mathrm{ha}^{-1}$; os teores de nitrogênio, até a concentração de $31 \mathrm{~g} \mathrm{~kg}^{-1}$ nas folhas de alho, amostradas no estádio de diferenciação floral ou 15 dias após esta fase, resultaram em maior produtividade total e comercial; a adubação nitrogenada de cobertura, em plantas de alho livre de vírus, proporcionou maior produtividade total e comercial sem ocorrência de superbrotamento.

\section{AGRADECIMENTOS}

À CAPES pela concessão da bolsa de mestrado à primeira autora e à empresa Genove Agronegócios pela estrutura e apoio técnico para o desenvolvimento do experimento.

\section{REFERÊNCIAS}

AGRIANUAL-Anuário Agrícola Brasileiro. 2007. São Paulo: Instituto FNP. 520p.

BÜLL LT; BERTANI RMA; VILLAS BÔAS RL; FERNANDES DM. 2002. Produção de bulbos e incidência de pseudoperfilhamento na cultura do alho vernalizado em função de adubações potássicas e nitrogenadas. Bragantia 61: 247-255.

BÜLL LT; VILLAS BÔAS RL; FERNANDES DM; BERTANI RMA. 2001. Fertilização potássica na cultura do alho vernalizado. Scientia Agricola 58: 157-163.

COSTA TMP; SOUZA JR; SILVA AM. 1993. Efeitos de diferentes lâminas de água e doses de nitrogênio sobre a cultura do alho (Allium sativum L.) cv. Juréia. Ciência e Prática 7: 239-246.

EMBRAPA. 1999. Sistema Brasileiro de Classificação de Solos. Brasília: Embrapa Produção de Informações. 412 p.

FERREIRA DF. 2003. Sisvar versão 4.2. Lavras: UFLA.

FILGUEIRA FAR. 2000. Novo manual de olericultura. Viçosa: UFV. 402p.

LIMA CP. 2005. Medidor de clorofila na avaliação de nutrição nitrogenada na cultura do alho vernalizado. Botucatu: UNESP-FCA. 95p (Tese mestrado).

LIPINSKI V; GAVIOLA HERAS S; FILIPPINI MF. 1995. Effect of irrigation, nitrogen fertilization and close size on yield and quality of coloured garlic (Allium sativum L.). Ciencia del Suelo 13: 80-84.

MALAVOLTA E. 1996. É uma verdade - o nitrogênio é exigido pelas plantas. Tradução e adaptação do original "It's the Truth-Nitrogen Is Required by Plants", PPI, Norcross, EUA. (Arquivo do Agronomo no 10).

MALAVOLTA E; VITTI GC; OLIVEIRA SA. 1997. Avaliação do estado nutricional das plantas: princípios e aplicações. Piracicaba: POTAFOS. 317p.

MAROUELLI WA; SILVA WLC; CARRIJO OA; SILVA HR. 2002. Produção e qualidade de alho sob regimes de água no solo e doses de nitrogênio. Horticultura Brasileira 20: 191-194.

PATEL BG; KHANAPARA VD; MALAVIA DD; KANERIA BB. 1996. Performance of drip and surface methods of irrigation for garlic (Allium sativum L.) under varying nitrogen levels. Indian Journal of Agronomy 41: 174-176.

RAIJ BV; ANDRADE JC; CANTARELLA H; QUAGGIO JA. 2001. Análise Química para avaliação da fertilidade de solos tropicais. Campinas: Instituto Agronômico. 285p.

RAIJ BV; CANTARELLA H; QUAGGIO JA; FURLANI AMC. 1996. Recomendações de adubação e calagem para o estado de São Paulo. Campinas: Instituto Agronômico. 285p. (Boletim técnico, 100).

RESENDE FV; FAQUIN V; SOUZA RJ. 2000. Efeito da adubação nitrogenada no crescimento e na produção de alho proveniente de cultura de tecidos e de multiplicação convencional. Revista Brasileira de Ciência do solo 24: 49-57.

RESENDE GM; SOUZA RJ. 2001a. Doses e épocas de aplicação de nitrogênio sobre a produtividade e características comerciais de alho. Horticultura Brasileira 19: 126-129.

RESENDE GM; SOUZA RJ. 2001b. Efeitos de tipos de bulbos e adubação nitrogenada sobre a produtividade e características comerciais do alho cv. "Quitéria". Horticultura Brasileira 19: 320-323.

RESENDE GM; SOUZA RJ; LUNKES JA. 1993. Influência do nitrogênio e paclobutrazol em alho cv. Quitéria. Horticultura Brasileira 11: 126-128.

RESENDE M. 1994. Uso de nível de clorofila na folha como indicador do momento de se aplicar nitrogênio em cobertura. Boletim Informativo da SBCS 19: 113.

SADARIASG; MALAVIADD; KHANPARAVD; DUDHATRA MG; VYAS MN; MATHUKIA RK. 1997. Irrigation and nutrient requirement of garlic (Allium sativum L.) under south Saurashtra region of gujarat. Indian Journal Agricultural Sciences 67: 402-403.

SYSTAT SOFTWARE. 2008. Systat: programas Sigmaplot e Sigmastat. Disponível em: www. systat.com . Acesso em:12 ago 2008.

TAIZ L; ZEIGER E. 2004. Fisiologia Vegetal. Porto Alegre: Artmed. 719p.

VERMA DP; SHARMA BR; CHADHA APS; BAJPAI HK; BHADAURIA UPS. 1996. Response of garlic (Allium sativum L.) to nitrogen, phosphorus and potassium levels. Advances in Plant Sciences 9: 37-41. 